\title{
Diseño e implementación de un sistema de control y monitoreo basado en HMI-PLC para un pozo de agua potable
}

\section{Design and Implementation of Control and Monitoring Systems Based on HMI-PLC for Potable Water Well}

\author{
Quezada-Quezada José Carlos \\ Área Académica de Ingeniería \\ Escuela Superior de Tizayuca \\ Universidad Autónoma del Estado de Hidalgo \\ Correo:jcarlos@uaeh.edu.mx \\ Flores-García Ernesto \\ Área Académica de Ingeniería \\ Escuela Superior de Tizayuca \\ Universidad Autónoma del Estado de Hidalgo \\ Correo:efloresg@uaeh.edu.mx
}

\author{
Bautista-López Jorge \\ Universidad Autónoma del Estado de México \\ Axapusco, Estado de México \\ Correo:jorgeb@uaem.edu.mx \\ Quezada-Aguilar Víctor \\ Área Académica de Ingeniería \\ Escuela Superior de Tizayuca \\ Universidad Autónoma del Estado de Hidalgo \\ Correo:vqua@hotmail.com
}

Información del artículo: recibido: enero de 2012, reevaluado: septiembre de 2012, aceptado: marzo de 2013

\section{Resumen}

Este trabajo se enfoca al diseño e implementación en un banco de pruebas de un sistema de control y monitoreo de descarga de agua en un pozo de agua potable, utilizando equipo especializado para automatización. Se diseñan las interfaces gráficas de usuario (Graphical User Interface, GUI) para interactuar con el operador. La interfaz hombre-máquina (Human Machine Interface, HMI) se implementó en software propietario y contempla reglas para control y monitoreo de las condiciones del sistema para el operador. La HMI se interconecta con un controlador lógico programable (Programmable Logic Controller, PLC) donde se implementan las reglas de protección del proceso en diagrama escalera (Ladder Diagram, LD).

\section{Descriptores:}

- control supervisorio y adquisición de datos (SCADA)

- controlador lógico programable (PLC)

- diagrama escalera (LD)

- interfaz gráfica de usuario (GUI)

- interfaz hombre máquina (HMI) 


\begin{abstract}
This project reports on the design and implementation in a workbench of a control and monitoring system of the discharge of water of a well. Graphic User's Interfaces (GUI) are designed for interaction with the operator. The Human Machine Interface (HMI) was implement in proprietor software and it contemplates the rules for control and monitoring of the conditions of the system for the operator, the HMI is also interconnected a Programmable Logic Controller (PLC) where the rules of protection of the process are implemented in Ladder Diagram (LD).
\end{abstract}

\section{Keywords:}

- Supervisory Control and Data Acquisition (SCADA)

- Programmable Logic Controller (PLC)

- Ladder Diagram (LD)

- Graphical User Interface (GUI)

- Human Machine Interface (HMI)

\section{Introducción}

En la industria, numerosos sistemas de manufactura y procesos continuos requieren máquinas y/o plantas productivas típicamente controladas mediante algoritmos generados en controladores lógicos programables (PLC), pudiendo ser modificados ante nuevos requerimientos de las máquinas o plantas de manera fácil, rápida y segura (Ljungkrantz et al., 2009; Gulpanich et al., 2005) mediante el uso de PLC, existen aplicaciones desde el control de motores hasta sistemas de control inalámbrico (Ahmed y Soo, 2009; Alheraish et al., 2006; Johnson, 2008). Los algoritmos de control desarrollados en PLC deben asegurar la confiabilidad del proceso mediante reglas que permitan incluir "todas" las posibilidades de riesgo, tanto para las personas como para la planta misma (Konaka et al., 2003; Devinder et al., 2005). Para este proyecto se ha desarrollado la lógica de control en diagrama escalera (LD) por ser todavía una de las más comúnmente utilizadas en los PLC y por consiguiente en el control de procesos (Johnson, 2008; Konaka et al., 2003).

Por otra parte, las interfaces humano máquina (HMI), ejecutadas en una computadora personal (PC), en la actualidad se utilizan para representar de forma "idéntica" la realidad de los procesos, permitiendo a los operadores una interrelación de los equipos físicos de la planta con los equipos virtuales de las interfaces gráficas de usuario (GUI) (Devinder et al., 2005; Mathiesen et al., 2006). Además, las HMI permiten incluir eventos (principalmente a través del ratón de la PC, mediante los comandos de configuración "mouse-down" y "mouse$\left.u p^{\prime \prime}\right)$ para realizar acciones de control y protección de los equipos del sistema, así como "leer" información de las variables de los PLC (mediante el acceso a las me- morias donde se encuentra el estado de las variables del proceso) y poder utilizar el estado de esas variables para indicar, mediante cambios de color en los equipos virtuales, la condición que guardan en el proceso real y facilitar al operador del sistema la toma de decisiones (Hall et al., 2002).

La integración HMI-PLC conforma un típico sistema de Control supervisorio y adquisición de datos (SCA$\mathrm{DA})$, que incluye interfaces de operación, sistemas de comunicación y equipo de instrumentación y control (Mathiesen et al., 2006), y la organización de estándares internacionales (ISO) ha desarrollado un modelo de interconexión de sistemas abiertos (OSI) con el objetivo de proveer una estructura de trabajo común a los desarrolladores de equipo de control y monitoreo de procesos (Clarke y Reynders, 2004).

El presente trabajo muestra al PLC como el sistema de control del proceso, a la HMI como el monitor del mismo incluyendo a las GUI diseñadas, a la integración HMI-PLC como el sistema de control y monitoreo del pozo de agua potable y las pruebas realizadas a éste. El desarrollo del sistema se plantea con una lógica de "estado de las variables-evento a realizar: protección y/o animación mediante color".

\section{PLC como controlador del sistema}

Para el diseño del algoritmo de control se han considerado los siguientes aspectos (Ashour, 2004; Barret, 2008):

1. Requerimientos generales del sistema de agua potable: variables a controlar y/o monitorear

2. Dispositivos de control utilizados: PLC, Sensores y Variador de velocidad "driver" 
3. Interconexión del sistema

4. Desarrollo del algoritmo de control y acondicionamiento de señales para monitoreo en LD.

\section{Requerimientos del sistema}

La función básica del sistema es extraer agua potable de un pozo para abastecer al municipio de Zapotlán de Juárez, Hgo., por lo que se requiere controlar la velocidad del conjunto motor-bomba y medir propiedades del agua a través de variables como: temperatura, $\mathrm{pH}$, concentración de cloro y conductividad en tiempo real; así como la presión en la tubería de descarga, lo cual permita asegurar el cuidado de las instalaciones, pero sobre todo, garantizar la calidad del agua.

\section{PLC, sensores y variador de velocidad}

El PLC utilizado es de tipo modular (Bolton, 2006; Domingo et al., 2003), con capacidad de módulos de entradas y salidas de señales digitales a 127 VCA y módulos de entradas y salidas de señales analógicas de 4 a $20 \mathrm{~mA}$, teniendo al sistema como se muestra en la figura 1.
Las entradas digitales se direccionan mediante \%I, las entradas analógicas por \%AI, las salidas digitales por $\%$ y las salidas analógicas por \%AQ. Las señales internas de memoria del PLC se direccionan con \%M para las señales digitales y con $\% \mathrm{R}$ para los registros de 16 bits (Fanuc, 2002).

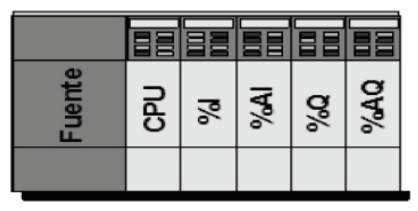

Figura 1. Hardware del PLC

La declaración de variables físicas y su respetivo direccionamiento en el PLC se muestran en la tabla 1.

A las variables que son requeridas en las GUI para su manipulación y/o monitoreo se les asigna el mismo TAG o etiqueta, y se asocian a la dirección correspondiente en la HMI. La asignación del TAG está en función del estándar de la Sociedad de Instrumentistas de América (Instrument Society of America, ISA, 1992).

La tabla 2 muestra las características de los equipos propuestos para la medición de las variables analógicas.

\begin{tabular}{ccc}
\hline Dirección & TAG & Descripción \\
\hline$\%$ I1 & HS_Físico & Paro de emergencia físico \\
$\%$ AI1 & TT & Señal de temperatura \\
$\%$ AI2 & C_AT & Señal de concentración de cloro \\
$\%$ AI3 & D_AT & Señal de pH \\
$\%$ AI4 & FT & Señal de flujo \\
$\%$ AI5 & PT & Señal de presión \\
$\%$ AI6 & CT & Señal de conductividad \\
$\% A I 7 ~$ & VEL_MB & Señal de velocidad del conjunto motor- \\
\%Q1 & A_P_MB & bomba \\
$\% A Q 1$ & SP_VELMB & Punto de consigna para velocidad de \\
& & conjunto motor-bomba \\
\hline
\end{tabular}

Tabla 1 Direccionamiento de variables en el PLC

\begin{tabular}{cccc}
\hline Variable & Intervalo* $^{*}$ & Intervalo $(\mathrm{SI})^{* *}$ & Principio \\
\hline Temperatura & $0-100^{\circ} \mathrm{C}$ & $273.15-373.15 \mathrm{~K}$ & RTD - Pt100 \\
Cloro & $0-250 \mathrm{mg} / \mathrm{L}$ & $0-250 \mathrm{Kg} / \mathrm{m}^{3}$ & Celula amperométrica \\
$\mathrm{pH}$ & $0-14$ & $0-14$ & Membrana de vidrio \\
Flujo & $0-50 \mathrm{~L} / \mathrm{s}$ & $0-0.05 \mathrm{~m}^{3} / \mathrm{s}$ & Magnético \\
Presión & $0-10 \mathrm{~kg} / \mathrm{cm}^{2}$ & $0-1 \times 10^{5} \mathrm{~Pa}$ & Fuelle $-\mathrm{LVDT}$ \\
Conductividad & $0-200 \mu \mathrm{S} / \mathrm{cm}$ & $0-2 \times 10^{-2} \mathrm{~S} / \mathrm{m}$ & Electrodos de titanio - en contacto \\
Velocidad & $0-1800 \mathrm{rpm}$ & $0-188.5 \mathrm{rad} / \mathrm{s}$ & Señal del controlador de velocidad \\
\hline
\end{tabular}

Tabla 2 Características de instrumentos de medición

\footnotetext{
* Mediciones con unidades utilizadas en la práctica por el operador del sistema

** Mediciones con unidades del sistema internacional (SI)
} 
Interconexión del sistema

La figura 2 muestra la conexión de los equipos de medición; HMI-PLC, PLC-Trasmisores de las variables, PLC-Controlador de velocidad, incluyendo la señal de paro de emergencia y de arranque y paro del conjunto motor-bomba.

\section{Diagrama escalera}

La figura 3 presenta el control del conjunto motorbomba, incluyendo las señales de protección y de alarmas del sistema. Cuando es activada la señal de paro de emergencia HS_FÍSICO o la señal de paro de emergencia virtual HS_SISTEMA (desde las GUI), el control lógico coloca a 0 (cero) la señal AUX_A_P_MB para que en las GUI se restablezcan las condiciones de animación del conjunto motor-bomba en estado de apagado. También, la señal del selector virtual D_F_ SISTEMA (dispositivo virtual en las GUI) inhabilita el encendido del motor; este dispositivo es propuesto para condiciones de mantenimiento del sistema de agua potable.

La figura 4 muestra el acondicionamiento de la variable temperatura para su monitoreo en las GUI. El acondicionamiento se propone para obtener una mayor precisión en las mediciones al utilizar valores reales en los indicadores virtuales en las GUI. El acondicionamiento es similar para las demás variables, con la dirección de registro distinta y con factores de conversión que corresponden al rango de medición de la variable en cuestión.

\section{HMI como monitor del sistema}

La GUI se desarrolló en software propietario para el diseño de HMI con conexión de comunicación de RS232 (PC) a RS485 (PLC).

El sistema se conforma por dos GUI; la PRINCIPAL, que muestra en su semejanza al sistema de agua potable con los respectivos equipos e instrumentos de medición, y la de MONITOREO, que muestra la lectura de las variables del sistema, incluyendo la velocidad del conjunto motor-bomba. La tabla 3 muestra los puntos de configuración declarados y habilitados en la HMI con su dirección asociada correspondiente para ser utilizados en las acciones de control, monitoreo y animación del sistema en las GUI. La figura 5 muestra el diagrama de flujo general que representa la configuración entre la HMI y el PLC.

Las figuras 6 y 7 muestran la GUI PRINCIPAL y la GUI de MONITOREO en modo de edición. En ambas GUI se tienen los elementos de protección, paro de emergencia físico mostrado mediante un letrero de seguridad al centro, paro de emergencia virtual representado mediante una palanca y dos letreros del estado que guarda la variable, selector virtual para la condición de dentro-fuera del sistema, y el botón de arranque-paro del conjunto motor-bomba.

La pantalla PRINCIPAL muestra de forma virtual el proceso real del sistema de agua potable; el conjunto motor-bomba de extracción de agua del pozo con su botón de arranque y paro y el variador de velocidad, los instrumentos utilizados en línea para cada una de las variables a monitorear, mostrando la GUI una ventana de medición para cada variable, respectivamente.

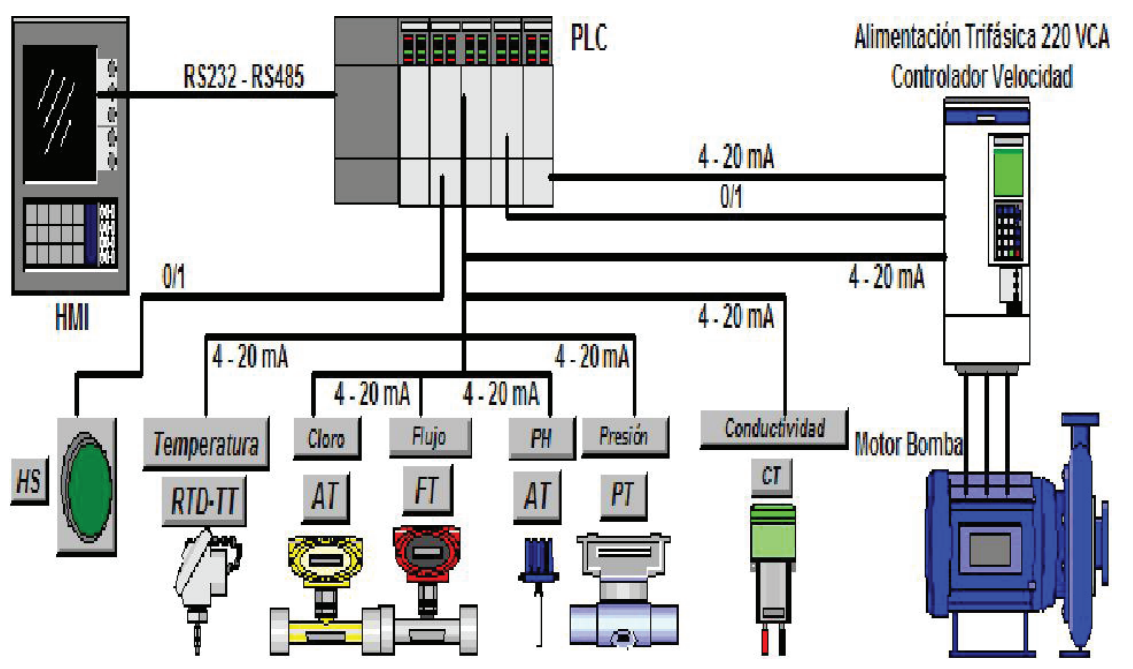

Figura 2. Interconexión del sistema 


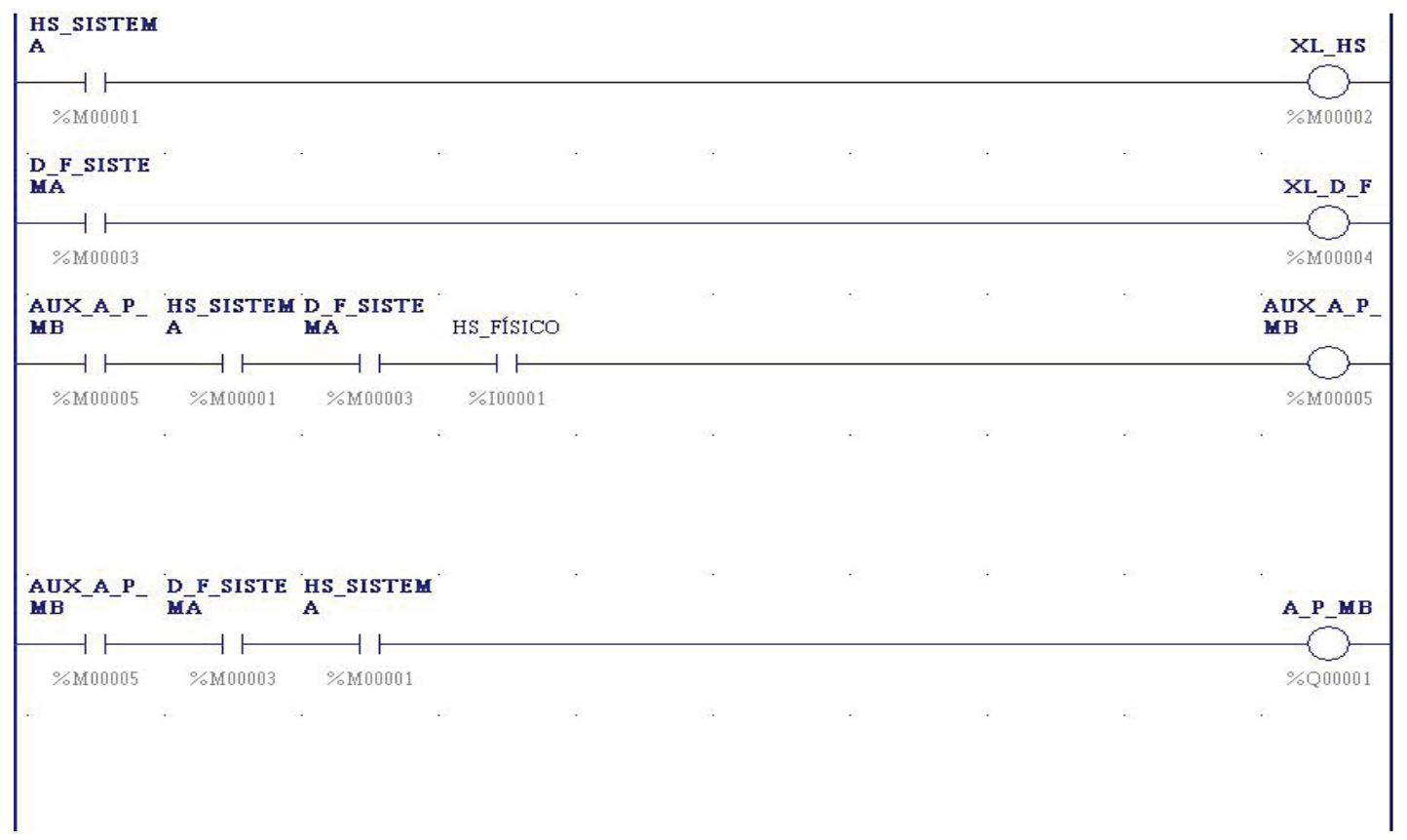

Figura 3. Diagrama escalera del control del sistema

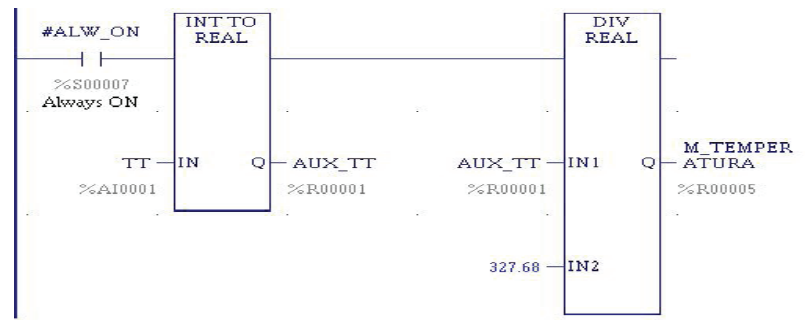

Figura 4. Diagrama escalera para acondicionamiento de señal
Tabla 3 Declaración de puntos de configuración de la HMI para control, monitoreo y animación

\begin{tabular}{lc}
\hline \multicolumn{1}{c}{ Punto en HMI } & Dirección en PLC \\
\hline HS_FÍSICO & $\% \mathrm{I} 1$ \\
HS_SISTEMA & $\% \mathrm{M} 1$ \\
XL_HS & $\% \mathrm{M} 2$ \\
D_F_SISTEMA & $\% \mathrm{M} 3$ \\
XL_D_F & $\% \mathrm{M} 4$ \\
AUX_A_P_MB & $\% \mathrm{M} 5$ \\
A_P_MB & $\% \mathrm{Q} 1$ \\
M_TEMPERATURA & $\% \mathrm{R} 5$ \\
M_CLORO & $\% \mathrm{R} 15$ \\
M_PH & $\% \mathrm{R} 25$ \\
M_FLUJO & $\% \mathrm{R} 35$ \\
M_PRESIÓN & $\% \mathrm{R} 45$ \\
M_CONDUCTIVIDAD & $\% \mathrm{R} 55$ \\
M_VELOCIDAD & $\% \mathrm{R} 65$ \\
VEN_TEMPERATURA & $\% \mathrm{M} 10$ \\
VEN_FLUJO & $\% \mathrm{M} 11$ \\
VEN_CLORO & $\% \mathrm{M} 12$ \\
VEN_PH & $\% \mathrm{M} 13$ \\
VEN_PRESIÓN & $\% \mathrm{M} 14$ \\
VEN_CONDUCTIVIDAD & $\% \mathrm{M} 15$ \\
VEN_VELOCIDAD & $\% \mathrm{M} 16$ \\
SP_VELMB & $\% \mathrm{AQ1}$ \\
\hline
\end{tabular}




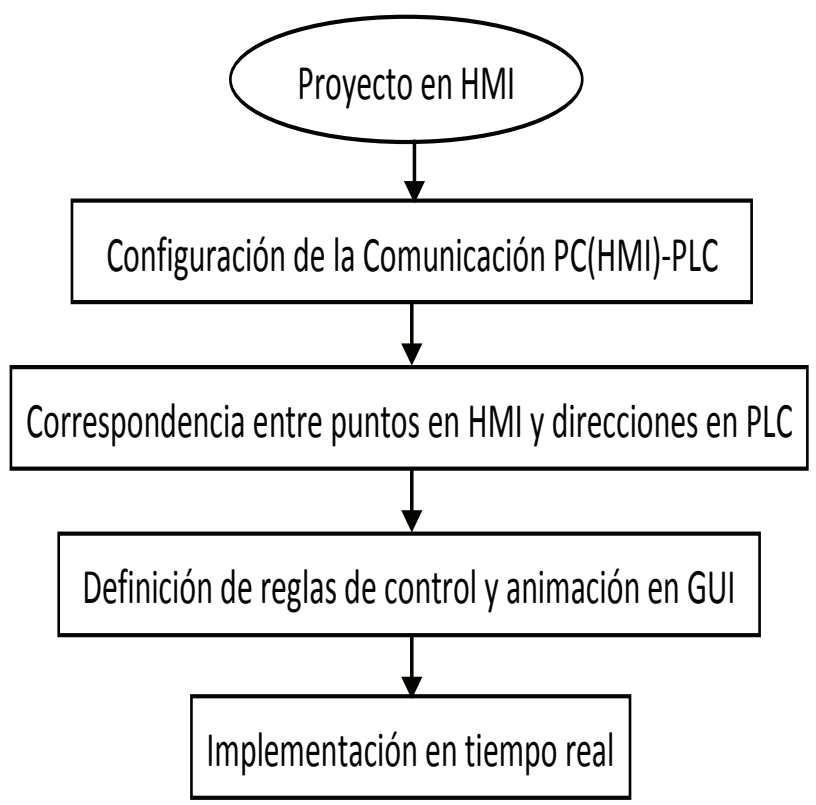

Figura 5. Diagrama de flujo para configuración de proyecto HMI-PLC

\section{Reglas de control}

La lógica ha sido desarrollada en una conjunción de la programación de estados de las variables y de la programación de eventos para realizar una acción (Cote et al., 2005). La figura 8 muestra el diagrama de eventos en las GUI y los estados de las variables en el PLC de las protecciones del sistema: HS_FÍSICO, HS_SISTEMA y D_F_SISTEMA.

La $\uparrow$ indica la ejecución de una acción con el mouse de la computadora en los instrumentos virtuales de las GUI.

Con base en las reglas de control, el arranque y paro del conjunto motor-bomba se puede realizar si HS_ FÍSICO, HS_SISTEMA y D_F_SISTEMA son igual a 0 (cero).

\section{Reglas para el monitoreo}

Para visualizar las ventanas auxiliares de monitoreo de las variables es necesario seleccionar el instrumento correspondiente y dar clic con el mouse. Para la GUI PRINCIPAL sólo puede estar una ventana auxiliar abierta a la vez. La GUI MONITOREO está configurada para visualizar todas las mediciones del sistema. La figura 9 muestra la ventana auxiliar de la variable temperatura; y el diagrama de eventos y estados de los puntos y variables en el PLC, respectivamente (figura 10).

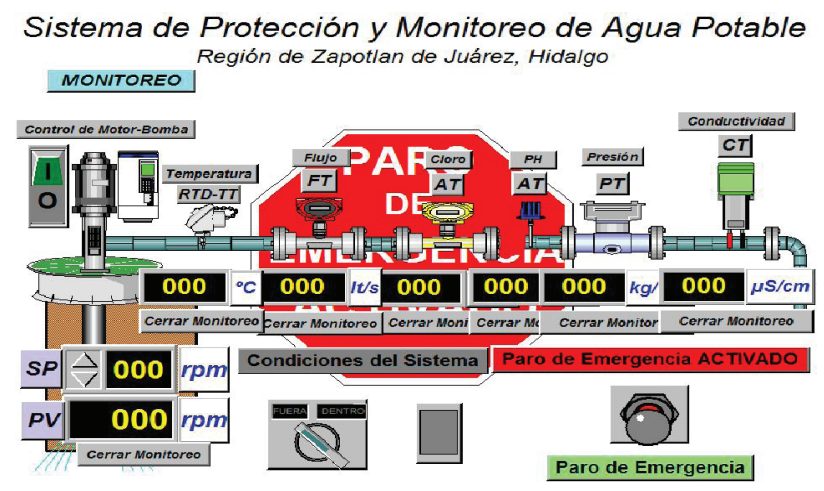

Figura 6. Interfaz gráfica PRINCIPAL en modo de edición

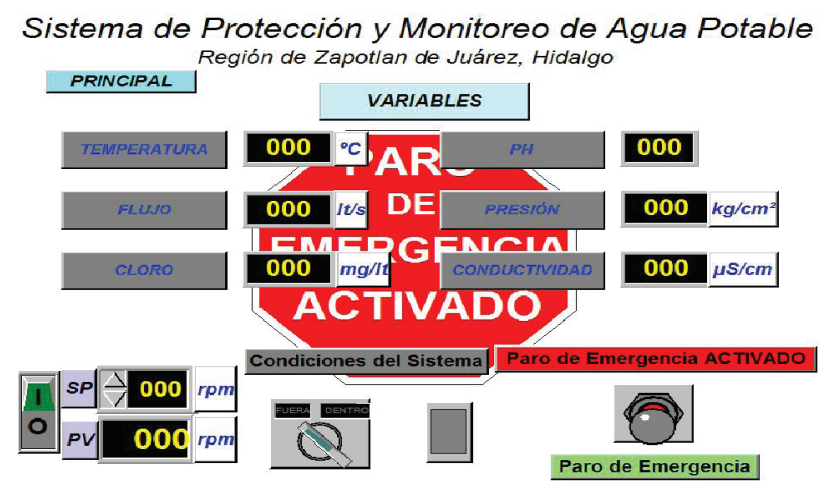

Figura 7. Interfaz gráfica de MONITOREO en modo de edición. La pantalla MONITOREO muestra la medición en tiempo real de todas las variables.

Para el control de velocidad del conjunto motor-bomba, se diseñó una ventana que permite, desde las GUI, manipular el punto de ajuste (Set Point, SP) de la velocidad deseada y a la vez monitorear la velocidad real del motor. La figura 11 muestra dicha ventana y la figura $12 \mathrm{su}$ diagrama de eventos.

Sólo cuando la ventana auxiliar de velocidad está activa se puede manipular el SP mediante el mouse en los botones tipo punta de flecha para incrementar o decremento de la variable.

\section{Pruebas del sistema de control y monitoreo}

A cada regla de control, monitoreo y animación de color, implementadas en el PLC y en las GUI desarrolladas, se les realizaron pruebas de funcionamiento. Para fines de explicación, en este trabajo se dividieron en tres tipos de pruebas al sistema:

1. Control y protecciones del sistema

2. Control de velocidad del conjunto motor-bomba

3. Monitoreo de las variables. 


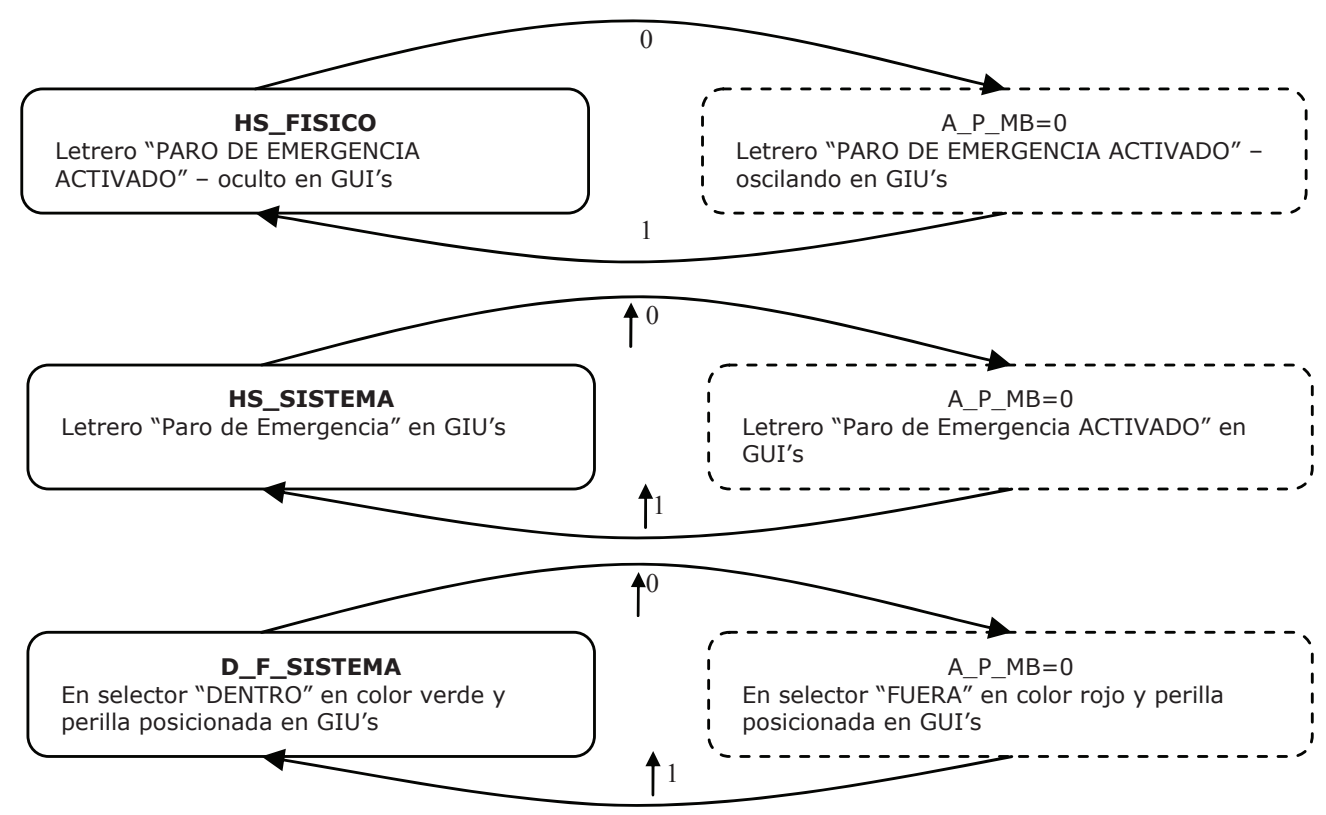

\section{$000{ }^{\circ} \mathrm{C}$}

Cerrar Mon itoreo
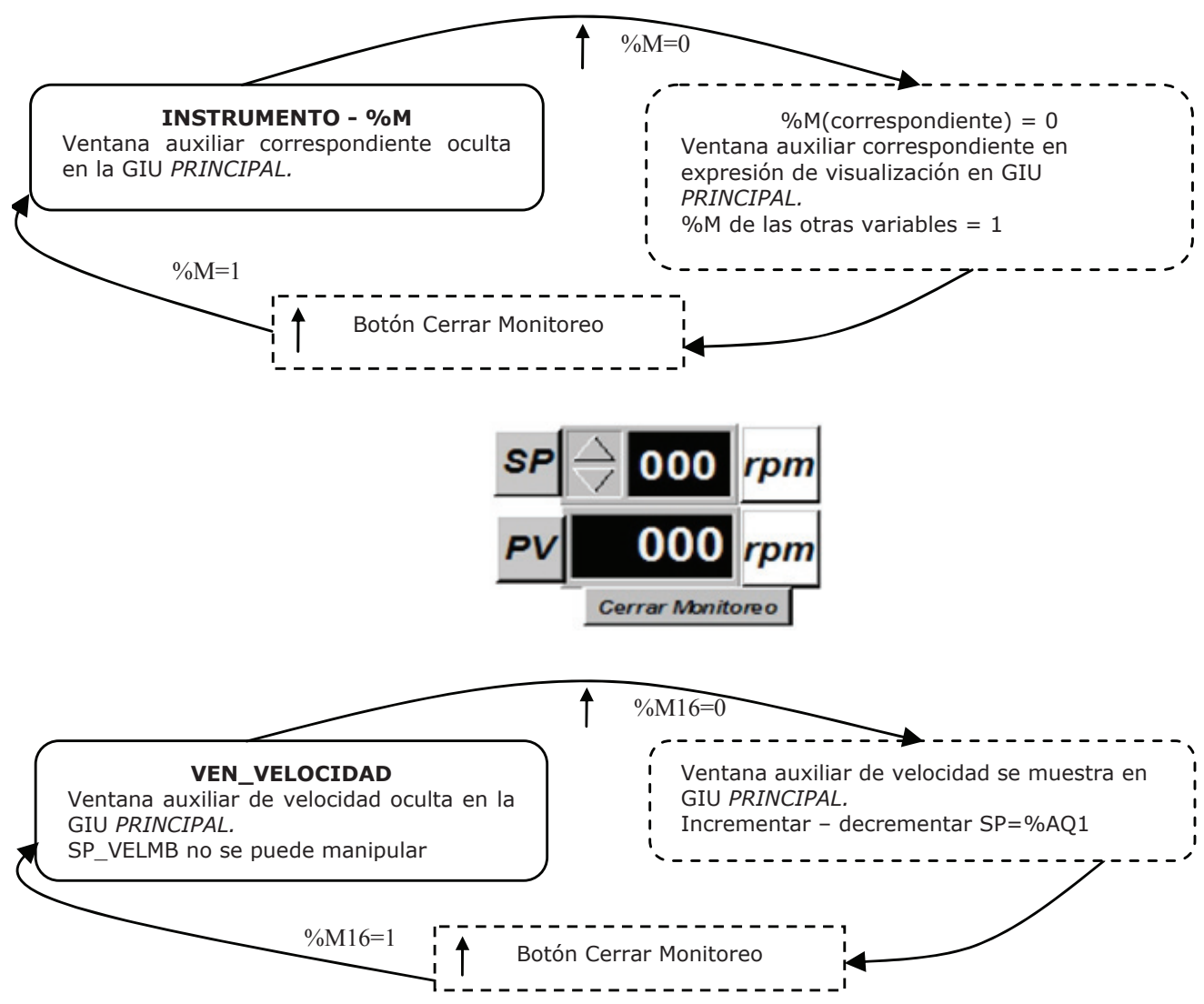

Figura 8. Diagrama de eventos en las GUI y estados de las variables en el PLC de las protecciones

Figura 9. Ventana auxiliar para monitoreo de la variable temperatura

Figura 10. Diagrama de eventos y estados para monitoreo de variables

Figura 11. Ventana de SP y monitoreo de velocidad del motor

Figura 12. Diagrama de eventos y estados de la velocidad del motor 


\section{Control y protecciones del sistema}

La figura 13 muestra a la GUI con el HS_FÍSICO=0, lo cual origina que el letrero de "PARO DE EMERGENCIA ACTIVADO" se muestre en las GUI, y al motor virtual en color rojo y al botón virtual de arranque-paro del motor en condiciones iniciales para su arranque.

La figura 14 muestra la condición de D_F_SISTE$\mathrm{MA}=0$ y HS_SISTEMA $=0$, donde el selector virtual es posicionado en "FUERA" con palabra en color rojo, así como el botón de alarma correspondiente. El interruptor virtual que representa a la palanca de paro de emergencia del sistema se mueve geométricamente a la posición inferior y cambia a color rojo, además de mostrarse la leyenda "Paro de Emergencia ACTIVADO".

La figura 15 muestra la ventana auxiliar de velocidad activada e indicando el SP deseado, así como el monitoreo de la velocidad real del conjunto motorbomba. La ventana auxiliar de velocidad del motor puede estar o no activa cuando se realiza el monitoreo

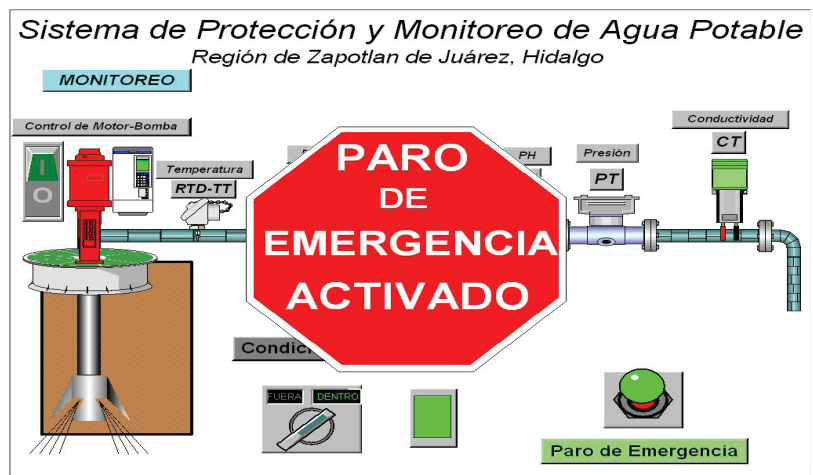

Figura 13. Sistema con HS_FíSICO=0

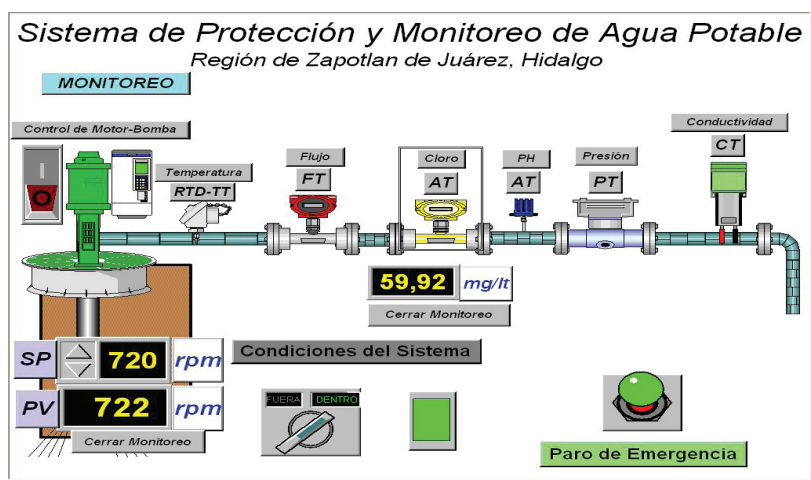

Figura 15. Ventana auxiliar de monitoreo de la variable temperatura de otra variable del proceso (temperatura, flujo, concentración de cloro, $\mathrm{pH}$, presión o conductividad).

La figura 16 muestra el monitoreo de la variable temperatura, condicionando a que sólo la ventana auxiliar para dicha medición esté presente; asimismo, la figura 17 muestra el monitoreo de la variable de presión.

La GUI de MONITOREO en tiempo real (figura 18), permite visualizar todas las variables de medición, incluyendo la ventana auxiliar de velocidad para su control, así como los instrumentos de protección del sistema del pozo de agua potable. Es importante señalar que al momento de la realización de la prueba mostrada, la medición de $\mathrm{pH}$ presenta valor nulo debido a que el circuito del sensor correspondiente estaba abierto.

La figura 19 muestra la estación de trabajo donde fueron realizadas las pruebas en tiempo real al sistema; puede observarse la GUI PRINCIPAL en el monitor de la computadora, así como el PLC, el controlador de velocidad y los sensores-transmisores de temperatura y presión.

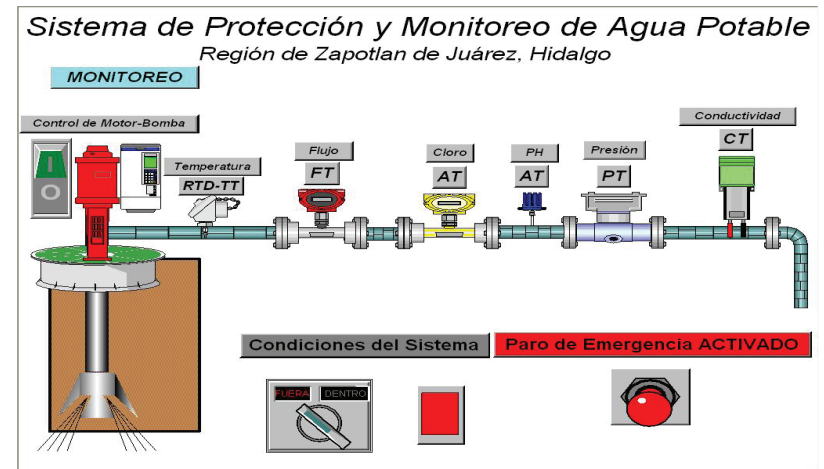

Figura 14. Sistema con HS_SISTEMA $=0$ y D_F_SISTEMA $=0$

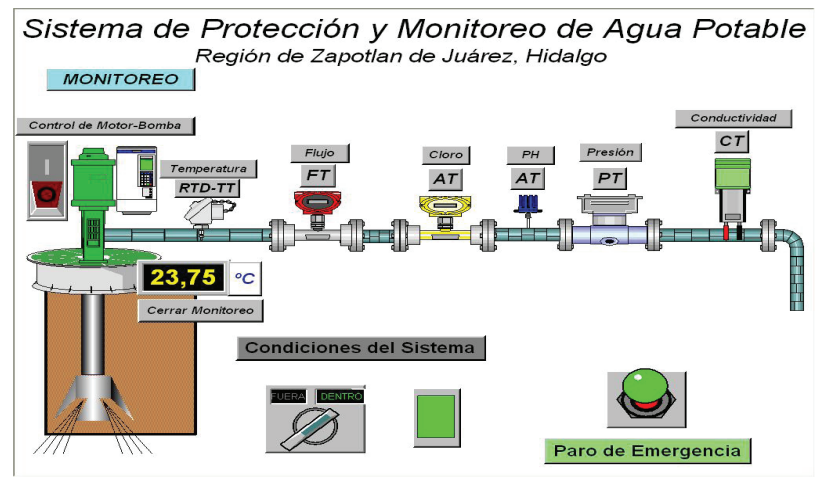

Figura 16. Ventana auxiliar de monitoreo de la variable temperatura 


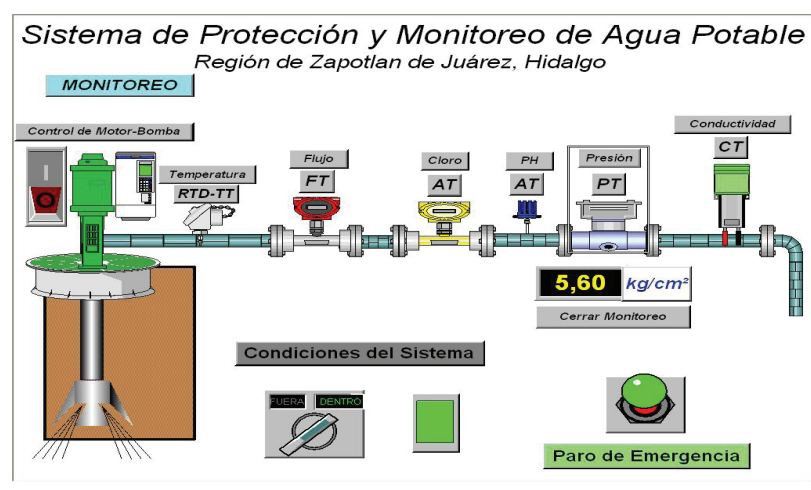

Figura 17. Ventana auxiliar de monitoreo de la variable presión

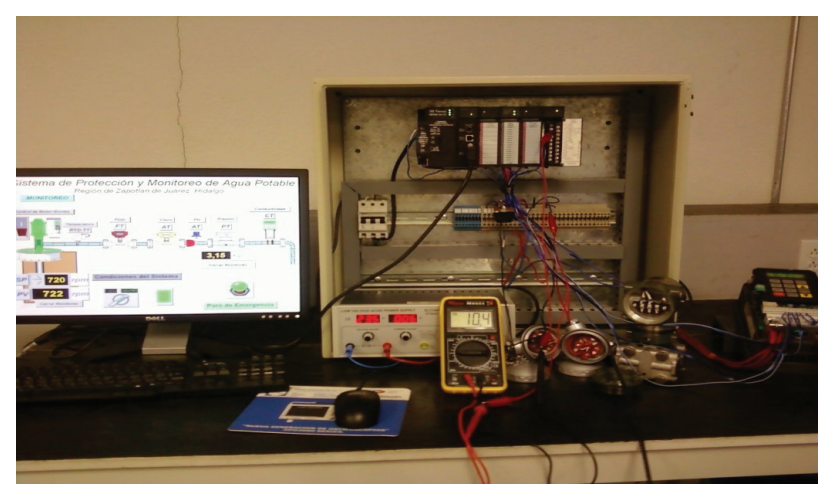

Figura 19. Banco de trabajo para pruebas del sistema en tiempo real

\section{Conclusiones}

Las tecnologías que más se utilizan para automatizar procesos continuos que requieren control con PLC, así como de la manipulación y del monitoreo de variables con posible animación en tiempo real a través de HMI, permiten apoyar a los operadores en la interpretación de los procesos y en la detección de fallas y la pronta solución de problemas en el sistema, mediante alarmas programadas; sin embargo, también traen como consecuencia la necesidad y los requerimientos de personal altamente calificado en éstas tecnologías para su aplicación, pero sobre todo, para desarrollar los algoritmos tanto de control y protección como los de aquellos eventos a realizarse en las GUI, las cuales también deben desarrollarse principalmente en similitud con la realidad de los sistemas y procesos a controlar.

\section{Agradecimientos}

Este trabajo ha sido apoyado por el PROMEP (Programa de Mejoramiento del Profesorado, México) a través

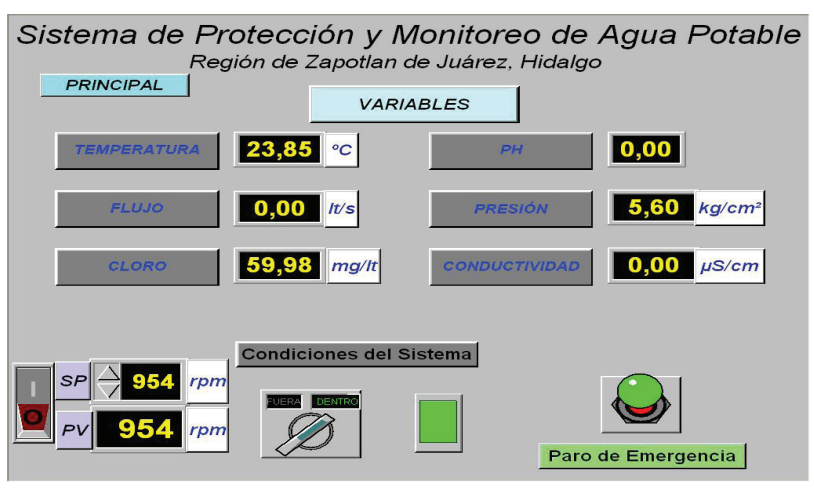

Figura 18. GUI de MONITOREO en tiempo real del proceso

del proyecto denominado: Sistemas de Monitoreo de Procesos en la Industria Química-Pozo de Agua Potable del Municipio de Zapotlán de Juárez, Hidalgo, México.

\section{Referencias}

Ahmed M.M. y Soo W.L. Customized SCADA System for Low Voltage Distribution Automation System, en: Transmission \& Distribution Conference \& Exposition: Asia and Pacific, octubre de 2009, pp.1-4.

Alheraish A., Alomar W., Abu-Al E.M. Programmable Logic Controller System for Controlling and Monitoring Home Application Using Mobile Network, en: Instrumentation and Measurement Technology Conference, IMTC 2006, Proceedings of the IEEE, abril de 2006, pp. 469-472.

American National Standard. Instrumentation Symbols and Identification, ANSI/ISA 5.1-1984 (R-1992), julio de 1992.

Ashour H. Automatic Transfer Switch (ATS) Using Programmable Logic Controller (PLC), Mechatronics, ICM ‘04, en: Proceedings of the IEEE International Conference, junio de 2004, pp. 531-535.

Barrett M. Managing the Invisible Assets. Engineering \& Technolo$g y$, volumen 3 (número 12), octubre de 2008: 50-52.

Bolton W. Programmable Logic Controllers, 4a ed., Elsevier, 2006, pp. 9-12.

Domingo J., Gámiz J., Grau A., Martínez H. Introducción a los autómatas programables, 1a ed., VOC, 2003, pp. 124, 135.

Clarke G. y Reynders D. Practical Modern SCADA Protocols: DNP3, 60870.5 and Related Systems, 1a. ed., Elsevier, 2004, pp. 4-5.

Cote D., St-Denis R., Kerjean S. Generative Programming for Programmable Logic Controllers, Emerging Technologies and Factory Automation, ETFA 2005, en: 10th IEEE Conference, volumen 2, septiembre de 2005, pp. 8, 748.

Devinder T., Suraj D., Gi-Nam W. Transformation from Petri Nets Model to Programmable Logic Controller Using One-to-One Mapping Technique, en: Computational Intelligence for Modeling, Control and Automation, 2005 and International Conference on Intelligent Agents, Web Technologies and Internet 
Commerce, International Conference, volumen 2, noviembre de 2005, pp. 228-233.

Fanuc G. ${ }^{\text {TM }}$. PLC Serie 90TM - 30/20/Micro, Juego de Instrucciones de la CPU, Manual de referencia GFK-0467-SP, 2002, pp. 2-10, 11, $27,31$.

Gulpanich S., Tipsuwanporn V., Suesut T., Tirasesth K. Implementation Programmable Logic Controller for THAILAND Industries, Computational Intelligence for Modeling, Control and Automation 2005 and International Conference on Intelligent Agents, Web Technologies and Internet Commerce, International Conference, volumen 2, noviembre de 2005, pp. 234-239.

Hall S., Cockerham K., Rhodes. What's your Color? [Human-Machine Interface Design]. Industry Applications Magazine, IEEE, volumen 8 (número 2), marzo-abril 2002: 50-54.

Johnson D. Programmable Logic Controllers. Control Engineering, septiembre de 2008, pp. 83-90.

Konaka E., Suzuki T., Okuma S. Safety Verification of Programmable Logic Controller Taking into Account the Physical Dynamics-Application to Material Handling Robots, en: SICE 2003 Annual Conference, volumen 1, agosto de 2003, pp. 818823.

Ljungkrantz O., Akesson K., Fabian M., Yuan C. Formal Specification and Verification of Industrial Control Logic Components.
IEEE Transactions Automation Science and Engineering, volumen 99, diciembre de 2009: 1-11.

Mathiesen M.L., Vefling H., Indergaard R., Aakvaag N. Trial Implementation of a Wireless Human Machine Interface to Field Devices, en: Emerging Technologies and Factory Automation, ETFA ‘06. IEEE Conference, septiembre de 2006, pp.189-193.

\section{Este artículo se cita:}

\section{Citación estilo Chicago}

Quezada-Quezada, José Carlos, Ernesto Flores-García, Jorge Bautista-López, Víctor Quezada-Aguilar. Diseño e implementación de un sistema de control y monitoreo basado en HMI-PLC para un pozo de agua potable. Ingeniería Investigación y Tecnología, XV, 01 (2014): 41-50.

\section{Citación estilo ISO 690}

Quezada-Quezada J.C., Flores-García E., Bautista-López J., Quezada-Aguilar V. Diseño e implementación de un sistema de control y monitoreo basado en HMI-PLC para un pozo de agua potable. Ingeniería Investigación y Tecnología, volumen XV (número 1), enero-marzo 2014: 41-50.

\section{Semblanza de los autores}

José Carlos Quezada-Quezada. Obtuvo el grado de maestro en ciencias en ingeniería mecatrónica en el Tecnológico de Estudios Superiores de Ecatepec, Ecatepec, Estado de México en el año 2008, y el título de ingeniero en electrónica por el Instituto Tecnológico de Lázaro Cárdenas, Michoacán, México en 1992. Ejerció profesionalmente en Fertilizantes Mexicanos, CFE, Siderúrgica Lázaro Cárdenas las Truchas, y en Fertilizantes Nacionales. Actualmente es profesor investigador de la Escuela Superior de Tizayuca de la Universidad Autónoma del Estado de Hidalgo, México. Entre sus principales intereses está la automatización de procesos continuos y máquinas sofisticadas mediante PLC, PAC y HMI.

Ernesto Flores-García: Obtuvo el grado de doctor y maestro en ciencias en la especialidad de control automático en el Centro de Investigación y de Estudios Avanzados, CINVESTAV-IPN, de la Ciudad de México, en 2011 y 2004, respectivamente. Recibió su título de ingeniería en aeronáutica por el Instituto Politécnico Nacional, IPN, de la Ciudad de México, en 2002. Sus temas de interés incluyen el control de sistemas electromecánicos, servomecanismos, robótica y microcontroladores. Ha ejercido como profesor de universidad en las áreas de control y matemáticas desde 2004. Actualmente es profesor investigador en la Universidad Autónoma del Estado de Hidalgo, México.

Jorge Bautista-López: Es candidato a maestro en ciencias en ingeniería en telecomunicaciones por la SEPI de la ESIME Zacatenco del IPN, México; posee el título de ingeniero en comunicaciones y electrónica por la ESIME Zacatenco, en 2002. Ejerció profesionalmente en Grupo 7 comunicación. Actualmente es docente del CU UAEM Valle de Teotihuacán y de la ESTi dependiente de la Universidad Autónoma del Estado de Hidalgo, impartiendo cátedra en las asignaturas de sistemas digitales, microprocesadores y redes de computadoras. Entre sus áreas de interés se encuentra la implementación de sistemas embebidos mediante Microcontroladores y PLC, y la propagación de señales inalámbricas.

Víctor Quesada-Aguilar: Realizó estudios de licenciatura en ingeniería industrial (1999-2004) y de maestría en ciencias en ingeniería industrial (2006-2008) en la Universidad Autónoma del Estado de Hidalgo, México. Ha laborado en el ámbito industrial y académico en instituciones tales como Unión de Crédito del Estado de Hidalgo, Procesos Electrolíticos SA, Colegio de Educación Profesional Técnica CONALEP Tizayuca, Instituto Educativo Cultural Hidalgo, Universidad Autónoma del Estado de Hidalgo (Preparatoria Efrén Rebolledo, Preparatoria Núm. 4 y Escuela Superior de Tizayuca). 